EPJ Web of Conferences 81, 05005 (2014)

DOI: $10.1051 /$ epjconf/20148105005

(C) Owned by the authors, published by EDP Sciences, 2014

\title{
Non-perturbative pion dynamics for the $\mathrm{X}(3872)$
}

\author{
V. Baru',2,a , E. Epelbaum ${ }^{1}$, A. A. Filin ${ }^{1}$, C. Hanhart ${ }^{3}$, A. E. Kudryavtsev², Yu. S. Kalashnikova ${ }^{2}$, \\ U.-G. Meißner ${ }^{3,4}$, and A. V. Nefediev ${ }^{2,5,6}$ \\ ${ }^{1}$ Institut für Theoretische Physik II, Ruhr-Universität Bochum, D-44780 Bochum, Germany \\ ${ }^{2}$ Institute for Theoretical and Experimental Physics, 117218 Moscow, Russia \\ ${ }^{3}$ Forschungszentrum Jülich, Institute for Advanced Simulation, Institut für Kernphysik (Theorie) and Jülich \\ Center for Hadron Physics, D-52425 Jülich, Germany \\ ${ }^{4}$ Helmholtz-Institut für Strahlen- und Kernphysik and Bethe Center for Theoretical Physics, Universität Bonn, \\ D-53115 Bonn, Germany \\ ${ }^{5}$ National Research Nuclear University MEPhl, 115409, Moscow, Russia \\ ${ }^{6}$ Moscow Institute of Physics and Technology, 141700, Dolgoprudny, Moscow Region, Russia
}

\begin{abstract}
We discuss the role of non-perturbative pion dynamics on the near-threshold resonant $\mathrm{X}(3872)$ charmonium state, which is assumed to be an S-wave $D \bar{D}^{*}$ bound system. We calculate the contribution to the width of the X(3872) from the $D \bar{D} \pi$ intermediate state treated non-perturbatively and compare it with different approximate approaches. Further, we explore the quark-mass dependence of the pole position of the $\mathrm{X}(3872)$ state. We find that the trajectory of the $\mathrm{X}(3872)$ depends strongly on the assumed quark-mass dependence of the short-range interactions which can be determined in lattice QCD calculations.
\end{abstract}

\section{Introduction}

After more than a decade after the discovery of the $\mathrm{X}(3872)$ by the Belle collaboration its nature still remains an open question, see Ref. [1] for a review. The resonance has the mass $M_{X}=(3871 \pm 0.17) \mathrm{MeV}$ and thus resides very close to the neutral $D \bar{D}^{*}$ threshold

$$
E_{B}=M_{D^{0}}+M_{\bar{D}^{* 0}}-M_{X}=(0.12 \pm 0.26) \mathrm{MeV} .
$$

It is therefore natural to assume that it has a large molecular admixture [2], see also Refs. [3, 4].

Recently, the quantum numbers of this state were determined by the LHCb Collaboration to be $1^{++}[5]$ which is consistent with its interpretation as an S-wave $D^{0} \bar{D}^{* 0} / \bar{D}^{0} D^{* 0}$ bound state, see e.g. [6, 7]. The small binding energy relative to the $D^{0} \bar{D}^{* 0}$ threshold allows for an effective field theory (EFT) formulation of the problem in analogy to the deuteron ${ }^{1}$. The pionless EFT framework based on pure contact $D \bar{D}^{*}$ interactions was first applied to the X(3872) in Ref. [8]. Due to the relevance of other dynamical scales, such a treatment is expected to be valid only in very narrow region around the threshold. In particular, the three-body neutral channel $\pi^{0} D^{0} \bar{D}^{0}$ opens at the energy $7 \mathrm{MeV}$ below the

\footnotetext{
ae-mail: vadimb@tp2.rub.de

${ }^{1}$ Implications of heavy quark and heavy flavour symmetries were utilised in Ref. [10] to predict partner states of the X(3872).
} 
$D^{0} \bar{D}^{* 0}$ threshold while the charged three-body channels $D^{ \pm} D^{\mp} \pi^{0}$ and $D^{+} \bar{D}^{0} \pi^{-} / D^{-} D^{0} \pi^{+}$reside about $2 \mathrm{MeV}$ above it. Furthermore, also the charged two-body channel $D^{c} \bar{D}^{* c}$ (with $c= \pm$ ) is located around $8 \mathrm{MeV}$ above the neutral channel. To incorporate the long-range pion physics, the so-called $\mathrm{X}$-EFT was developed in Ref.[9] based on the assumption that pions can be treated perturbatively. Recently, this framework was extended to include higher-order corrections and then used to predict the pion-mass dependence of the X-pole [11]. On the other hand, the perturbative treatment of pions is known to be not applicable in the deuteron channel [12] which shows certain similarities with the $\mathrm{X}(3872)$. The role of non-perturbative pions was investigated in many phenomenological studies, see e.g. Refs.[14-16], all of them however include one-pion-exchange (OPE) in the static limit, i.e. under the assumption that the D-mesons are infinitely heavy particles. Meanwhile, the pion in the $D \bar{D}^{*}$ potential can go on shell and thus the three-body $\pi D \bar{D}$ unitarity cuts should be taken into account ${ }^{2}$.

In this Contribution we discuss effects induced by the non-perturbative pion dynamics on the X(3872) state within the EFT framework, see Refs.[17, 18] for more details. In particular, we test the validity of the static OPE approximation for the partial decay width $X(3872) \rightarrow D \bar{D} \pi$ and study the dependence of the $\mathrm{X}$ binding energy on the light quark masses which is a precondition to extract a valuable information about the $D \bar{D}^{*}$ interactions from upcoming and ongoing lattice simulations.

\section{Formalism}

We solve a system of coupled-channels Faddeev-type three-body equations for the $D \bar{D} \pi$ system in the $J^{P C}=1^{++}$channel

$$
\begin{aligned}
& a_{00}^{n n^{\prime}}\left(\boldsymbol{p}, \boldsymbol{p}^{\prime}, E\right)=\lambda_{0} V_{00}^{n n^{\prime}}\left(\boldsymbol{p}, \boldsymbol{p}^{\prime}\right)-\sum_{i=0, c} \lambda_{i} \int \frac{d^{3} k}{\Delta_{i}(k)} V_{0 i}^{n m}(\boldsymbol{p}, \boldsymbol{k}) a_{i 0}^{m n^{\prime}}\left(\boldsymbol{k}, \boldsymbol{p}^{\prime}, E\right), \\
& a_{c 0}^{n n^{\prime}}\left(\boldsymbol{p}, \boldsymbol{p}^{\prime}, E\right)=\lambda_{c} V_{c 0}^{n n^{\prime}}\left(\boldsymbol{p}, \boldsymbol{p}^{\prime}\right)-\sum_{i=0, c} \lambda_{i} \int \frac{d^{3} k}{\Delta_{i}(k)} V_{c i}^{n m}(\boldsymbol{p}, \boldsymbol{k}) a_{i 0}^{m n^{\prime}}\left(\boldsymbol{k}, \boldsymbol{p}^{\prime}, E\right),
\end{aligned}
$$

where $\lambda_{i}$ stand for the known isospin coefficients and the OPE potential containing the three-body propagator at leading order reads ${ }^{3}$

$$
V^{n n^{\prime}}\left(\boldsymbol{p}, \boldsymbol{p}^{\prime}\right)=-g^{2} \frac{\boldsymbol{p}_{n}^{\prime} \boldsymbol{p}_{n^{\prime}}}{2 m+m_{\pi}+\frac{p^{2}}{2 m}+\frac{p^{\prime 2}}{2 m}+\frac{\left(\boldsymbol{p}+\boldsymbol{p}^{\prime}\right)^{2}}{2 m_{\pi}}-M-i 0 .} .
$$

Here, the indices $n, n^{\prime}$ are contracted with the corresponding indices of the $D^{*}$ polarisation vectors, $m, m_{*}$ and $m_{\pi}$ stand for the $D, D^{*}$ and the pion mass, in order, and the energy $E$ is defined relative to the neutral two-body threshold $M=m_{* 0}+m_{0}+E$. Furthermore, the strength of the potential $g$ is extracted from the decay width $D^{*} \rightarrow D \pi$, see e.g. Ref. [18] for a more extended discussion of the input quantities. The OPE potential (3) connects the four $D$-meson channels defined as $|0\rangle=$ $D^{0} \bar{D}^{* 0}, \quad|\overline{0}\rangle=\bar{D}^{0} D^{* 0}, \quad|c\rangle=D^{+} D^{*-}, \quad|\bar{c}\rangle=D^{-} D^{*+}$, and the amplitude $a_{0}=\left(a_{00}-a_{c 0}\right) / 2$ contains the relevant information about the X-pole. Note that the same three-body cut is also taken into account in the $D \bar{D}^{*}$ propagators $\Delta_{i}$ due to dressing $D^{*}$ by the self-energy $(\pi D)$ loops.

It should be stressed that in full analogy to the NN problem $[19,20]$, the OPE does not fall off at large momenta and thus requires renormalisation. The $D \bar{D}^{*}$ potential in an S-wave $\left(V^{S S}\right)$ is to be modified to include the contact interaction $C_{0}$

$$
V^{S S}\left(p, p^{\prime}\right) \rightarrow C_{0}+V^{S S}\left(p, p^{\prime}\right)
$$

\footnotetext{
${ }^{2}$ It is shown in Ref. [13] that the 3-body unitary cuts play the crucial role in the $D_{\alpha} \bar{D}_{\beta}$ system, if the $D_{\beta}$ width is dominated by the $S$-wave $D_{\beta} \rightarrow D_{\alpha} \pi$ decay.

${ }^{3}$ In Ref. [21] the role of relativistic corrections in the non-perturbative approach including 3-body effects was addressed.
} 
MESON 2014 $-13^{\text {th }}$ International Workshop on Production, Properties and Interaction of Mesons

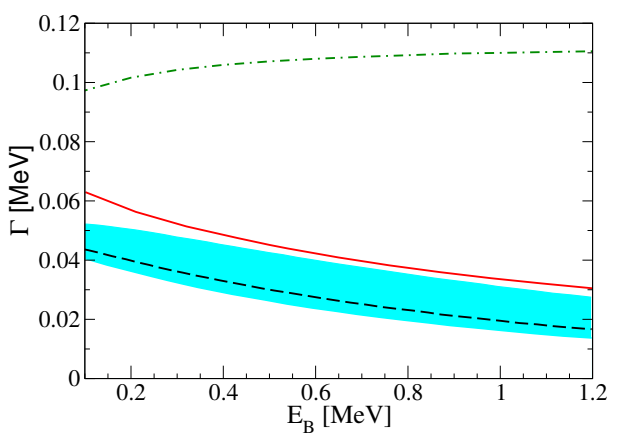

Figure 1. The $X$ width as a function of the binding energy $E_{B}$ for several different calculations: (i) solution of the problem with the non-perturbative OPE in the static limit - (green) dot-dashed line; (ii) solution of the full dynamical problem with non-perturbative OPE - (red) solid line; (iii) the X-EFT calculations: LO [6] - the dashed line and NLO [9] - the (blue) band.

For the sharp cut-off regularisation scheme used in our calculation $C_{0}(\Lambda)$ is adjusted to produce a bound state of the $X(3872)$ for any given cut-off $\Lambda$.

In order to analyse the light quark-mass dependence we allow all quantitates such as the $D$ and $D^{*}$-meson masses, the coupling constant and the pion decay constant to vary with $m_{\pi}$, i.e. we perform an expansion of all such quantities in terms of the parameter $\delta m_{\pi} / \mathrm{M}$ [18], where the small scale is the difference of the running and physical pion masses $\delta m_{\pi}=m_{\pi}-m_{\pi}^{\text {ph }}$, while the large scale M is given by a typical hadronic scale $\sim 1 \mathrm{GeV}$. In addition to OPE, also the contact term has to vary with $m_{\pi}$ to ensure that the binding energy $E_{B}\left(m_{\pi}\right)$ is approximately $\Lambda$-independent for the running pion mass. Assuming that the leading correction to the the physical-limit quantity $C_{0}^{\mathrm{ph}}(\Lambda)$ is analytic with the quark masses, we may write

$$
C_{0}\left(\Lambda, m_{\pi}\right)=C_{0}^{\mathrm{ph}}+\delta C_{0}=C_{0}^{\mathrm{ph}}(\Lambda)\left(1+f(\Lambda) \frac{m_{\pi}^{2}-m_{\pi}^{\mathrm{p}^{2}}}{\mathrm{M}^{2}}\right) .
$$

The leading $\Lambda$-dependence of the contact interaction is captured by $C_{0}^{\mathrm{ph}}(\Lambda)$, while the dimensionless function $f(\Lambda)$ absorbs the extra $\Lambda$-dependence which appears for values of the pion mass away from the physical point. Therefore, we fix the $\Lambda$-dependence of the contact interaction requiring that both the binding energy $E_{B}$ as well as its slope at the physical point, $\left.\left(\partial E_{B} / \partial m_{\pi}\right)\right|_{m_{\pi}=m_{\pi}^{\mathrm{ph}}}$, are $\Lambda$-independent.

\section{Discussion and conclusions}

First, we discuss the impact of non-perturbative pions on the decay width $X \rightarrow D \bar{D} \pi$ [17], as shown in Fig. 1. We find that the perturbative inclusion of pions is justified, while the static approximation with non-perturbative pions leads to a significant overestimation of this observable. Thus, we conclude that the appropriate treatment of the three-body dynamics is mandatory.

The pion mass dependence of the binding energy of the $\mathrm{X}(3872)$ is illustrated in Fig. 2. The trajectory of the $\mathrm{X}(3872)$ depends strongly on the assumed quark-mass dependence of the shortrange interactions which can be parametrized by the slope $\left.\left(\partial E_{B} / \partial m_{\pi}\right)\right|_{m_{\pi}=m_{\pi}^{\text {phys }}}$ and is, in principle, measurable in lattice QCD. This is demonstrated in Fig. 2 where the resulting pion mass dependence is shown for two different values of the slope of a natural size. The sizeable difference between the pion-full and pion-less approaches at higher values of the pion mass for positive values of the slope indicates the important role of pion dynamics in this scenario, see also Ref. [11] for an analogous study within the X-EFT ${ }^{4}$. These findings will be useful for chiral extrapolations of the future lattice-QCD

\footnotetext{
${ }^{4}$ Our results are in conflict with those of Ref. [22], the differences however can be traced back to conceptual problems of the approach used there, see Ref. [23] for a detailed discussion.
} 


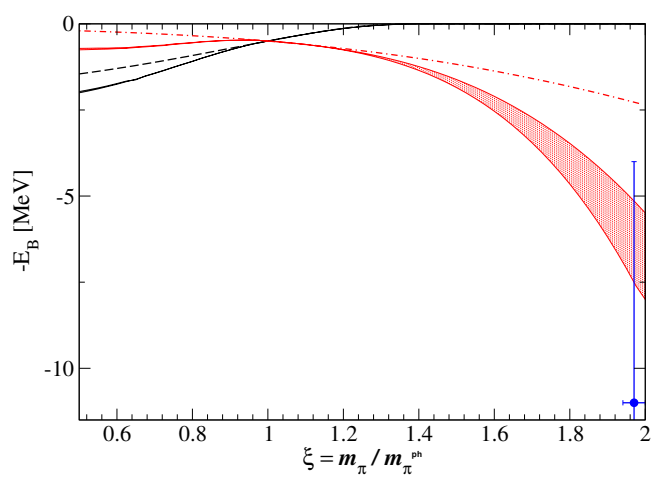

Figure 2. Pion mass dependence of the $X(3872)$ binding energy. The red filled band corresponds to the positive slope $\left.\left(\partial E_{B} / \partial m_{\pi}\right)\right|_{m_{\pi}=m_{\pi}^{\mathrm{ph}}}=0.7 \times 10^{-2}$ while the black filled band corresponds to the negative slope $\left.\left(\partial E_{B} / \partial m_{\pi}\right)\right|_{m_{\pi}=m_{\pi}^{\mathrm{ph}}}=-1.5 \times 10^{-2}$. In both cases, the ultraviolet cutoff in the integral equations is varied in the range $\Lambda \in[400,700] \mathrm{MeV}$. The dashed and dash-dotted curves represent the corresponding results of the pionless approach. The blue point depicts the first lattice calculation of the $\mathrm{X}(3872)$ [24].

results for the $\mathrm{X}(3872)$ binding energy (see Ref. [24] for the first results) and will provide insights into its binding mechanism once the value of the slope parameter is determined.

\section{Acknowledgements}

This work is supported in part by the DFG and the NSFC through funds provided to the Sino-German CRC 110, by the EU project HadronPhysics3 and the ERC project 259218 NUCLEAREFT.

\section{References}

[1] N. Brambilla et al., Eur. Phys. J. C 71, 1534 (2011).

[2] C. Hanhart et al., Phys. Rev. D 76, 034007 (2007).

[3] S. Weinberg, Phys. Rev. 130, 776 (1963).

[4] V. Baru et al., Phys. Lett. B 586, 53 (2004).

[5] Aaij et al. [LHCb Collaboration], Phys. Rev. Lett. 110, 222001 (2013).

[6] M. B. Voloshin, Phys. Lett. B 579, 316 (2004).

[7] E. Braaten and M. Lu, Phys. Rev. D 76, 094028 (2007).

[8] M. T. AlFiky, F. Gabbiani, and A. A. Petrov, Phys. Lett. B 640, 238 (2006).

[9] S. Fleming, M. Kusunoki, T. Mehen, and U. van Kolck, Phys. Rev. D 76, 034006 (2007).

[10] F.-K. Guo, C. Hidalgo-Duque, J. Nieves and M. P. Valderrama, Phys. Rev. D 88, 054007 (2013).

[11] M. Jansen, H.-W. Hammer and Y. Jia, Phys. Rev. D 89, 014033 (2014).

[12] S. Fleming, T. Mehen and I. W. Stewart, Nucl. Phys. A 677, 313 (2000).

[13] A. Filin et al, Phys. Rev. Lett. 105, 019101 (2010).

[14] N. A. Tornqvist, Phys. Lett. B 590, 209 (2004).

[15] C. E. Thomas and F. E. Close, Phys. Rev. D 78, 034007 (2008).

[16] Y.-R. Liu, X. Liu, W.-Z. Deng and S.-L. Zhu, Eur. Phys. J. C 56, 63 (2008).

[17] V. Baru et al., Phys. Rev. D 84, 074029 (2011).

[18] V. Baru et al., Phys. Lett. B 726, 537 (2013).

[19] G. P. Lepage, nucl-th/9706029.

[20] E. Epelbaum and U.-G. Meißner, Few Body Syst. 54, 2175 (2013).

[21] Yu. S. Kalashnikova and A. V. Nefediev, JETP Lett. 97, 70 (2013).

[22] P. Wang and X. G. Wang, Phys. Rev. Lett. 111, 042002 (2013).

[23] V. Baru, E. Epelbaum, A.A. Filin, F.-K. Guo, H.-W. Hammer, C. Hanhart, U.-G. Meißner and A.V. Nefediev, in preparation.

[24] S. Prelovsek and L. Leskovec, Phys. Rev. Lett. 111, 192001 (2013). 